\title{
Biomechanically-Informed Training: The Four Pillars for Knee and ACL Injury Prevention Built Upon Behavior Change and Motivation Principles
}

\author{
Cyril J. Donnelly ${ }^{1,2, *}$, Ben S. Jackson ${ }^{2}$, Daniel F. Gucciardi ${ }^{3}$ and Jeff Reinbolt ${ }^{4}$ \\ 1 Rehabilitation Research Institute of Singapore (RRIS), Nanyang Technological Institute, \\ Singapore 308232, Singapore \\ 2 School of Human Science (Exercise and Sport Sciences), The University of Western Australia, \\ Perth 6009, Western Australia, Australia; ben.jackson@uwa.edu.au \\ 3 School of Physiotherapy and Exercise Science, Faculty of Health Science, Curtin University, \\ Perth 6845, Western Australia, Australia; d.gucciardi@curtin.edu.au \\ 4 Department of Mechanical, Aerospace and Biomedical Engineering, University of Tennessee, \\ Knoxville, TN 37916, USA; reinbolt@utk.edu \\ * Correspondence: cyril.donnelly@ntu.edu.sg
}

Received: 19 May 2020; Accepted: 25 June 2020; Published: 28 June 2020

\begin{abstract}
Injury prevention frameworks are critical for preventing musculoskeletal injury and improving rehabilitation outcomes. However, their relative successes in translation arguably rely on two interlinked components: (1) the quality of the empirical evidence used to develop the intervention (content), and (2) the effective application of behavior change and motivation principles to optimise participant adherence and engagement (delivery). The purpose of this commentary is to develop an injury prevention and rehabilitation framework using the best available physics-based simulation, biomechanics, and behavior change research. The intervention as a whole is entitled biomechanically-informed training (BIT). While investigators have previously examined the relative merits of different training genres (e.g., plyometric, balance, resistance), what makes BIT novel is that it explicitly targets the biomechanical mechanisms that mitigate musculoskeletal injury risk (i.e., force) in ways that are underpinned by established behavior change principles. The four pillars of BIT refer to focused exercise use, irrespective of the training genre, to improve an individual's: (1) knee flexion dynamics, (2) dynamic trunk control, (3) gastrocnemius muscle strength, and (4) hip muscular strength. We also present experimental data from two independent training studies verifying the efficacy of BIT for the prevention of knee and anterior cruciate ligament (ACL) injury.
\end{abstract}

Keywords: self-determination theory; translation; prophylactic; rehabilitation; lower limb

\section{Background}

Technological advancements such as the invention of stop-action photography [1], the force platform [2], and the programmable computer have elevated the field of biomechanics as an important research discipline within the human sciences [3]. In parallel with improvements in motion capture technologies, musculoskeletal modelling, and human simulation, the field of biomechanics has become central to our understanding of the mechanical aetiology (external and internal forces) of musculoskeletal injury, re-injury, injury risk, re-injury risk, musculoskeletal disease initiation, and musculoskeletal disease progression. Although, the measurement of force at the level of the joint, muscle, tendon, and articular surfaces of the knee is technically challenging and heavily reliant on complex modelling of motion capture and medical imaging data is arguably why biomechanists have generally been non-central to the design, implementation, and assessment of prophylactic training 
interventions and neuromuscular rehabilitation programs. However, biomechanists must collaborate with coaches and clinicians to better understand the nuanced difference between establishing training programs based on scientifically proven principles (e.g., the program proposed in this paper), compared with intuitively designed programs.

It is not only the biomechanical underpinnings of a program that may shape injury prevention and rehabilitation outcomes. Regardless of the quality of program content, it is imperative that participants (e.g., athletes, patients) engage fully in a program in order to derive the intended benefits [4]. In this respect, behavior change principles and motivation theory play an important role in the application and successful delivery of empirically designed programs. Insight into the practical methods that can be used to support participant engagement (e.g., adherence, maintenance, motivation) in such programs is available in the literatures on behavior change techniques [5] and self-determination theory [6]. Nonetheless, despite the promise of these frameworks for supporting injury prevention program outcomes, to date they have arguably been underutilised in the development and implementation of prophylactic training interventions and rehabilitation research. In the material that follows, we will present the foundation of biomechanically-informed training (BIT), which is the program (e.g., four pillars), and the psychological and behavior change principles the program is built upon.

\section{The Context}

In Australia, a country with a population less than 25 million, one billion dollars is spent annually on lower limb musculoskeletal injury management [7-9]. Extrapolating this statistic to the United States, which has a population of approximately 328 million people, the annual cost of lower limb musculoskeletal injury management may be as high as 13 billion dollars. One lower limb injury that stands out among all others is a rupture to the anterior cruciate ligament (ACL). The reason ACL injuries receive such attention in the sport and rehabilitation literature is because they are associated with high health care costs, as well as short- and long-term health and quality of life issues for the injured/re-injured athlete. Management of an ACL injury is met with relatively high health care costs because surgical reconstructions are commonplace treatments [9], which are coupled with long rehabilitation periods (4 to 12 months) [10] and high rates of re-injury during recovery [11,12]. Short- and long-term health-related quality of life for those with ACL injuries is diminished as their physical activity levels are substantially reduce within two to three years of the injury event [13], and over $50 \%$ develop debilitating musculoskeletal disorders such as knee osteoarthritis within 10 years [14]. These populations are thus predisposed to inactivity, obesity, and obesity-related chronic health conditions like type 2 diabetes and cardiovascular disease in their later stages of life. It is particularly concerning that ACL injury rates appear to be increasing worldwide [15], and it is projected that ACL injuries will place an even greater burden on global health care costs and health-related quality of life issues in the near and distant future.

\section{The Problem}

Governments and health practitioners are aware of these ACL injury statistics, and substantial research attention has been devoted to lower limb and ACL injury/re-injury prevention as well as rehabilitation. Outcomes from this research have proven in principle that combinations of different training genres, such as balance, plyometric, resistance, and/or technique training can be used to reduce the lower limb and ACL injury risk among adolescent female populations ( $<18$ years) [16,17]. There is comparably little evidence supporting this generalised training approach for reducing injury risk among adult (male and female) sport-active populations. Two factors that have arguably not been well-developed nor implemented are 1) the biomechanical focus (and specificity) of the exercises (i.e., content) used within the intervention and can be a secondary focus to their development and implementation, and 2) athletes' experiences (e.g., adherence, motivation) in the intervention program are generally absent in their design and execution [18-22]. As a result, there is a need for injury prevention researchers to focus on how to maximise the training content and delivery 
methods of injury prevention and rehabilitation training protocols to translate lower limb and ACL injury/re-injury research more effectively among heterogeneous (young, adolescent, adult, male and female) sport populations.

\section{A Potential Solution}

The focus of this communication is to present the empirical evidence for the content and delivery of a novel lower limb injury/re-injury prevention training protocol for all athletic populations. In general, lower limb injury prevention training interventions have been developed and implemented without a clear mechanistic (e.g., biomechanical/neuromuscular) understanding of how the prescribed exercises within the intervention reduce injury risk during sport and exercise. Further, lower limb injury prevention research has yet to embrace behavioral science (e.g., communication, training, instructional) principles that support motivation and adherence among program participants as core considerations (Figure 1). To highlight the efficacy of BIT in reducing lower limb and ACL risk/rates, we will present two case examples.

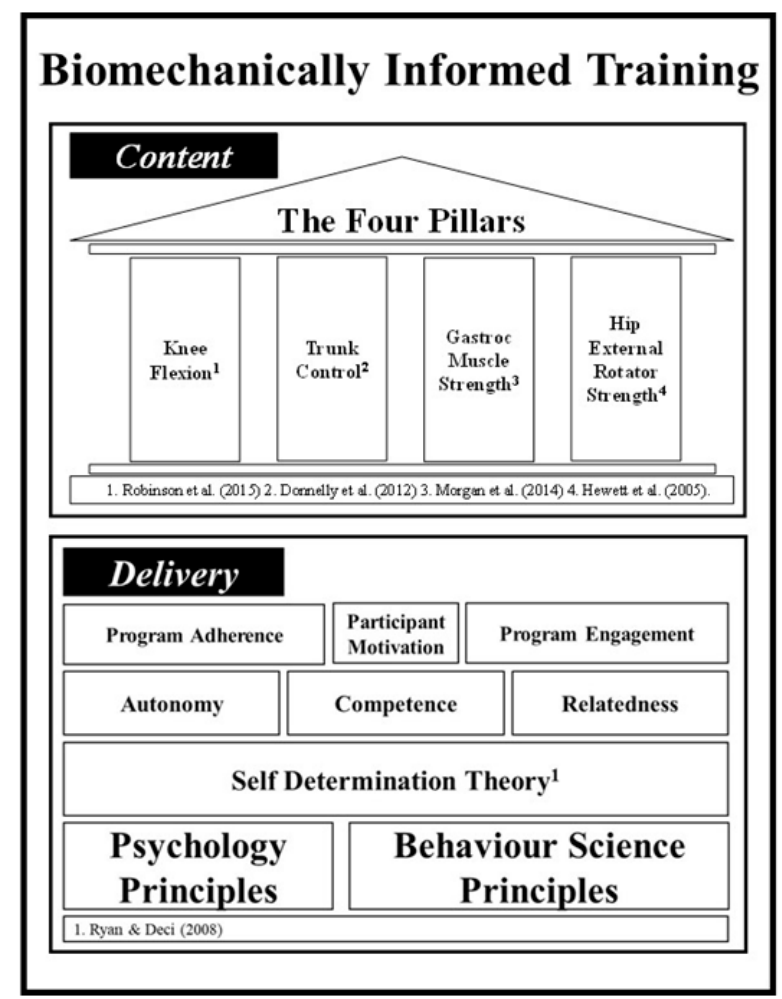

Figure 1. Theoretical overview of biomechanically-informed training (BIT). The empirical evidence for the training content above and the psychological and behavior change foundation for the delivery methods below.

\section{Biomechanically-Informed Training}

The content of BIT is based on best practice physics-based simulation and laboratory-based biomechanics research. What makes the content of this training approach novel is the explicit focus on targeting the mechanisms that mitigate injury risk (i.e., relationship between movement, joint loading, and injury risk). With the majority of sport participation and recreational lower limb injuries occurring at recreational levels [8], the exercises used within the training content have been purposely designed to be body weight-based (examples of trialed exercises can be found at www.youtube.com/bodyfitworkouts). This consideration is important because the training content can be implemented in a broad range of training environments (regardless of skill level), and does not require specialized equipment. 
These considerations abate potential barriers that may prevent the seamless integration of BIT within existing training/exercise regimes or environments.

The focus on delivery methods within the program refers to the integration of practical strategies designed to support participant adherence, maintenance, and motivation, that are drawn from established behavior change and motivation literature. It is important to draw from our understanding of motivational processes to structure program delivery in a way that promotes and maintains participant enjoyment and motivation. Such strategies are well-documented in the self-determination theory literature (e.g., [6]), and are essential given the established association between participant motivation and the success of health promotion programs (e.g., [4,23]). The application of self-determination theory principles to injury prevention and rehabilitation is not new, but these methods have debatably been underutilised in the context of lower limb and ACL injury/re-injury prevention protocols. The value of self-determination theory lies, in part, in the practical information that it provides to help programmers create environments that support participants' basic psychological needs for autonomy, competence, and relatedness [6]. We will 1 outline specific approaches to support these needs in injury prevention settings later in this communication; however, it is important to note here that the creation of 'need-supportive' environments often relies in large part on the communication and delivery styles adopted by program instructors (for a review, see [24]). The purposeful consideration and injection of instructional strategies to support participants' psychological needs in the design and implementation of lower limb injury prevention is likely to optimise positive downstream effects on participants' motivation, engagement, and the overall prophylactic effects of the program. These motivationally-enriched strategies can be augmented by an extensive and diverse range of behavior change techniques that can be customised to the individual needs of participants (for reviews, see $[24,25])$.

\section{Program Content}

The four pillars of the biomechanically-informed training content are built upon foundational biomechanics research focused on understanding the principles and mechanisms of movement and how it relates to ACL and re-injury. The four pillars or training messages forged from this research are to improve athletes':

- knee flexion dynamics [26],

- trunk control [27],

- gastrocnemius muscle strength [28], and

- hip external rotator strength during dynamic sporting tasks [29].

\subsection{Training Content Pillar \#1: Knee Flexion Dynamics}

Based on a novel probabilistic modelling approach, vector-field analysis [30], Robinson et al. [26] used a canonical correlation analysis (CCA) which is the vector-field equivalent of linear regression to show that an athlete's knee flexion angle during the impact phase of unanticipated stepping is significantly related $(p=0.011)$ to non-sagittal plane knee moments (Figure 2) [26]. Though it may seem intuitive that knee flexion angle would be related to an individual's risk of injury in sport, a biomechanical link between sagittal plane knee kinematics and peak knee moments known to elevate knee and ACL injury risk in sport had yet to be established in the sport biomechanics literature. These results therefore provide the empirical evidence needed to support the clinical recommendation to increase knee flexion angle at the impact phase (0 to 30\% stance) of unanticipated sidestepping tasks where non-contact ACL injury risk is arguably the greatest [15]. 

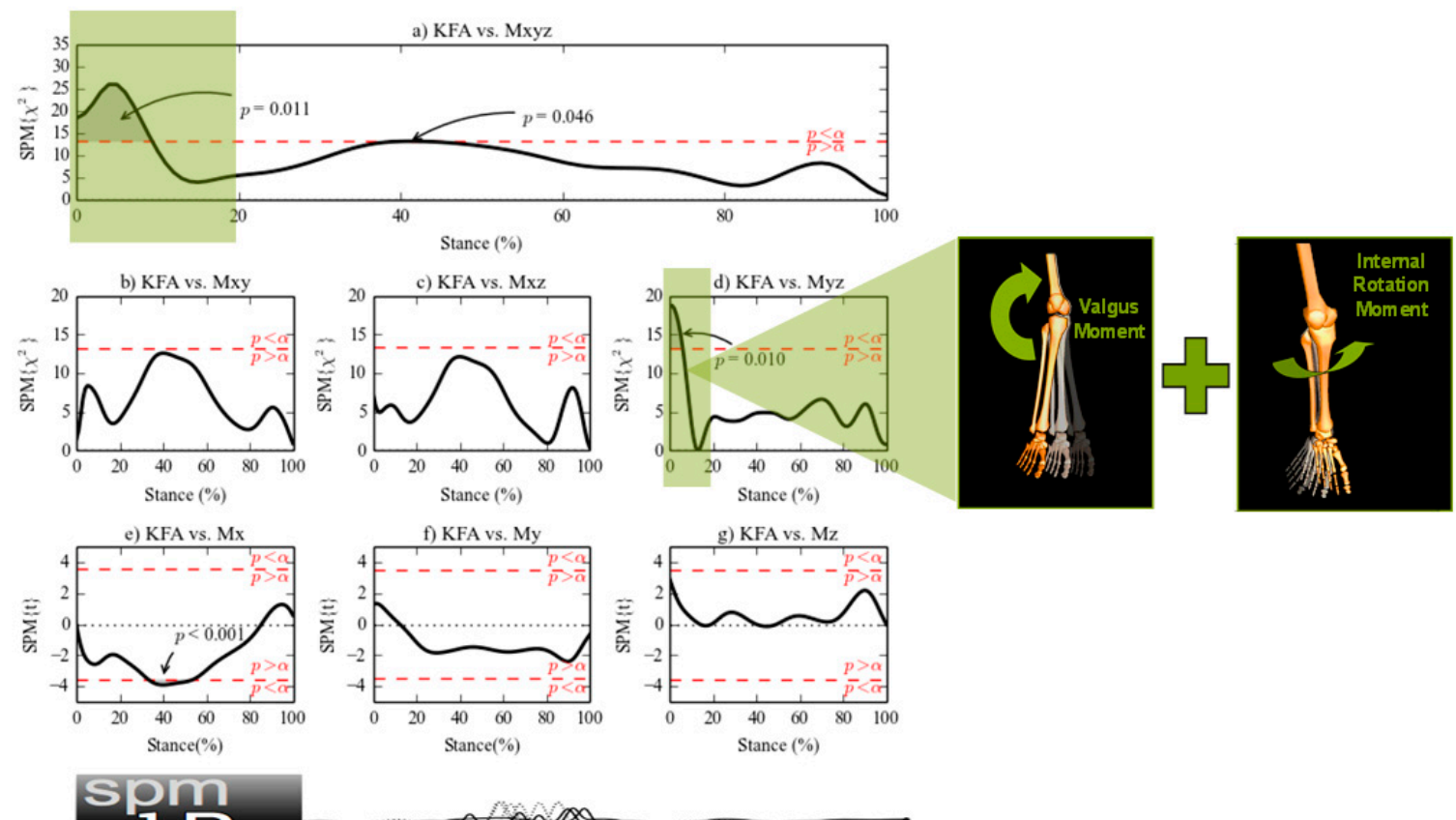

Figure 2. The top pane shows the relationship between knee flexion and three-dimensional knee moments $(\mathrm{Mx}, \mathrm{y}, \mathrm{z})$. This relationship was primarily explained by combined frontal (valgus) and transverse plane (internal rotation) knee moments (middle), not the individual knee moment components (bottom).

\subsection{Training Content Pillar \#2: Trunk Control}

To appreciate fully how simulation research can be used to answer mechanistic research questions associated with injury/re-injury, one must first understand how the Newton-Euler equations of motion are used to define human movement. Briefly, a simulation of human motion is defined using the Newton-Euler equations of motion (1): Where $\ddot{q}$ is the generalised coordinate accelerations due to joint torques, $\tau$, Coriolis, and centrifugal forces, $C(q, \dot{q})$, as a function of generalised coordinates, $q$, and their velocities, $\dot{\mathrm{q}}$, gravity, $\mathrm{G}(\mathrm{q})$, and external forces applied to the model, $\overrightarrow{\mathrm{F}}$, and its inverse of the mass matrix, $[\mathrm{M}(\mathrm{q})]^{-1}$, which contains segment mass' and inertial properties. As observed within the Newton-Euler equations of motion, a relationship exists between the joint angles $\left(\iint \ddot{q}\right)$ and joint torques $(\tau)$ to create a forward dynamic simulation. Through the manipulation of this computational relationship, which is founded in the laws of physics, researchers/clinicians are capable of changing the way an individual moves in-silico to produce a new subject-specific movement pattern associated with reduced injury risk and re-injury risk, and/or mitigate disease initiation/progression.

$$
\ddot{\mathrm{q}}=[\mathrm{M}(\mathrm{q})]^{-1}\{\tau+\mathrm{C}(\mathrm{q}, \dot{\mathrm{q}})+\mathrm{G}(\mathrm{q})+\overrightarrow{\mathrm{F}}\}
$$

Within the musculoskeletal modelling and simulation framework OpenSim (simTk.org) [31], Donnelly et al. [27] developed computational methods capable of optimizing an athletes' movement patterns during change of direction sporting tasks in attempts to reduce their risk of ACL injury during unplanned sidestepping. The optimised movement strategy was to control the position and acceleration of their whole body centre of mass during change of direction sporting tasks. By redirecting an athlete's whole body centre of mass by just $4 \mathrm{~cm}$ in their intended direction of travel (i.e., medially), an athlete can expect to reduce their peak abduction knee moments and ACL injury risk by approximately $40 \%$, which is a fantastic return on investment (Figure 3) [27]. As the trunk segment weighs over one-half of an individual's total mass, the dynamic control of this segment, above all others in the kinematic chain, is of paramount importance for reducing an athlete's risk of lower limb injury in sport. The applied 
clinical message from this research is that the dynamic control of the upper body during sporting tasks is important for reducing an athlete's lower limb injury risk in sport.

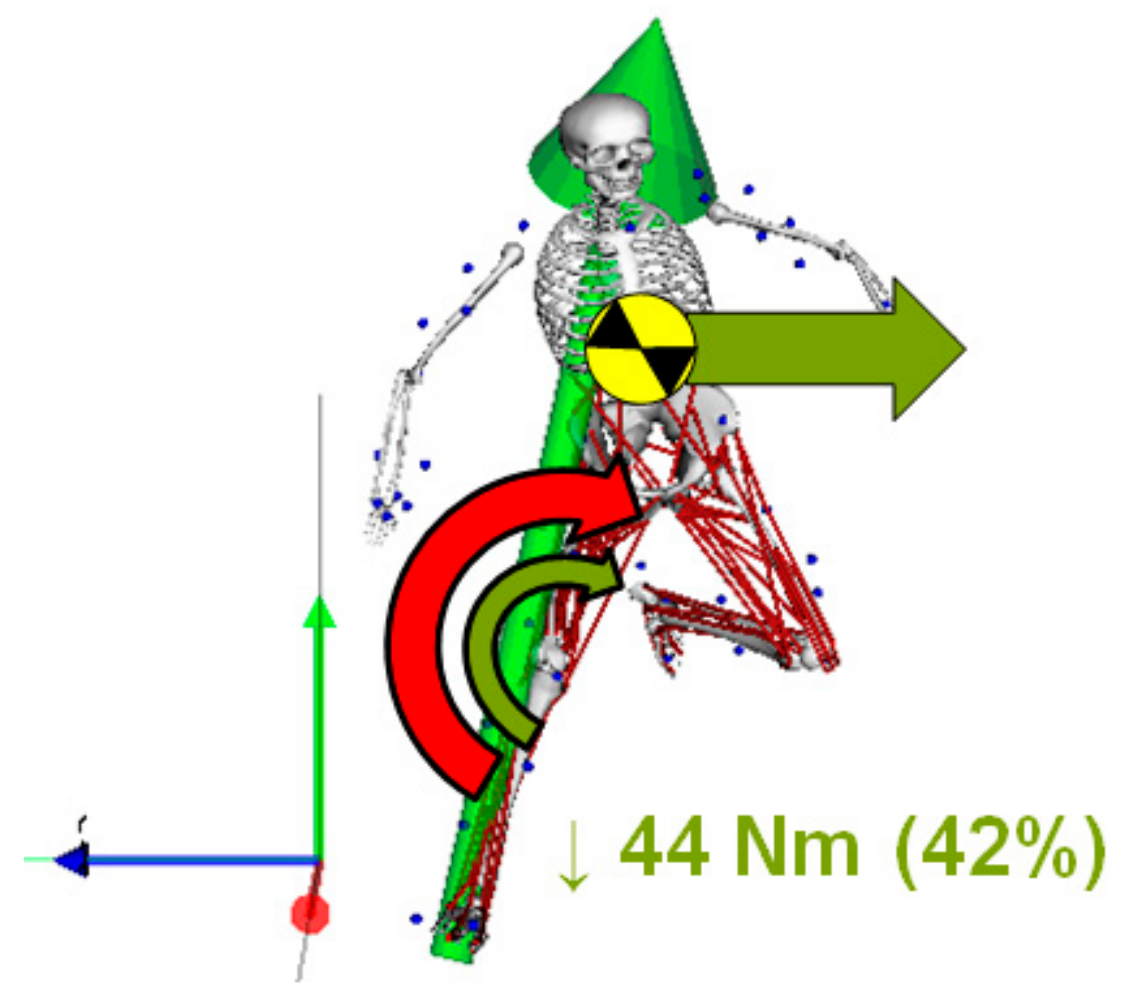

Figure 3. Overview of upper body mechanics and peak valgus knee moments during unplanned sidestepping.

\subsection{Training Content Pillar \#3: Gastrocnemius Muscle Strength}

Again, within the musculoskeletal modelling and simulation framework OpenSim (simTk.org), Morgan et al. [28] used computed muscle control (CMC) to calculate and characterise the muscle forces an athlete uses during single-leg landing sport tasks. The primary finding from this research was that the relative contribution of the gastrocnemius was elevated relative to the hamstrings during the impact phase of single-leg landing (Figure 4) [29], a sporting task where one-half of all non-contact ACL injuries are known to occur [32,33]. These findings represent a paradigm shift in the literature, challenging the views of over 30 years of clinical research, which have presumed that the hamstring muscles were the primary muscle group supporting the knee during landing. The mechanical model explaining these results are founded within established biomechanics research. The primary goal of the neuromuscular system during any task (e.g., standing, walking, landing) is to prevent the centre of mass from falling to the ground. Together with the quadriceps and hip extensors, the gastrocnemius muscles are used to generate support moments, which are moments of force counteracting the forces pulling you to the ground and allowing you to keep your body upright. Being a biarticular muscle, the gastrocnemius serves a secondary role, elevating joint compression during single-leg landing, which is used to stiffen and support the knee and ACL from the external forces and moments that can strain and rupture it. The applied clinical message from this research is that the gastrocnemius muscles play an important, underappreciated role in supporting the knee against externally applied knee loads during dynamic sporting tasks. 

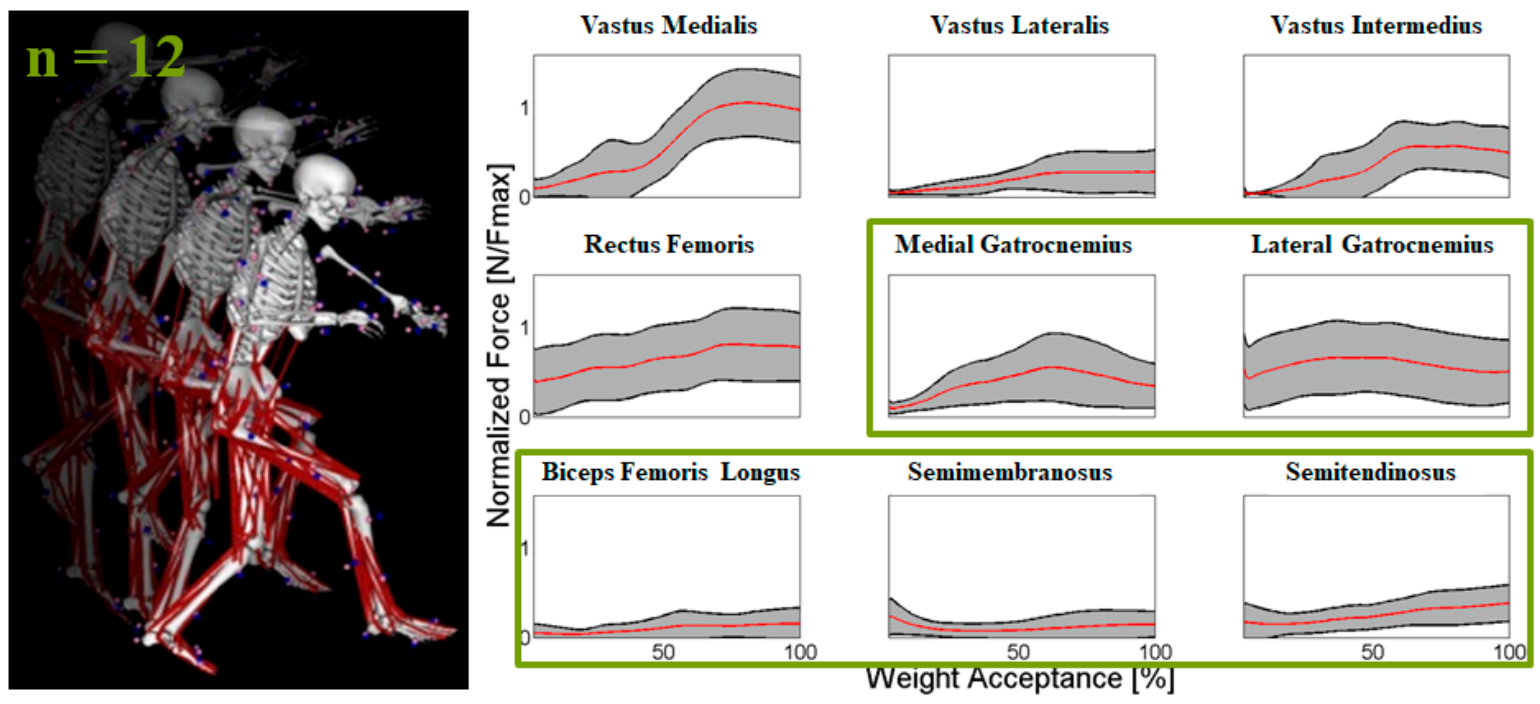

Figure 4. Overview of individual lower limb muscle contributions during single-leg landing.

\subsection{Training Content Pillar \#4: Hip External Rotator Strength}

Research by Hewett et al. [29] has shown that 'dynamic valgus' knee postures, or the 'inward buckling' (medial frontal plane shift) of the knee is predictive of ACL injury among adolescent female populations $\left(R^{2}=0.88\right)$ [29]. This 'dynamic valgus' posture is the result of excessive hip internal rotation (and adduction), combined with knee flexion (Figure 5). Consequently, the means by which an athlete attains a 'dynamic valgus' knee posture is likely attributed to poor hip neuromuscular control/strength during dynamic sporting tasks. This evidence has provided a rationale for coaches and clinicians to shift their focus above the knee, towards the hip joint, to better understand how the hip external rotator muscles (i.e., gluteals) function to prevent athletes from attaining these dangerous knee postures during dynamic sporting tasks. The applied clinical message from this research is not to forget about the hip musculature in supporting the knee against externally applied knee loads during dynamic sporting tasks.

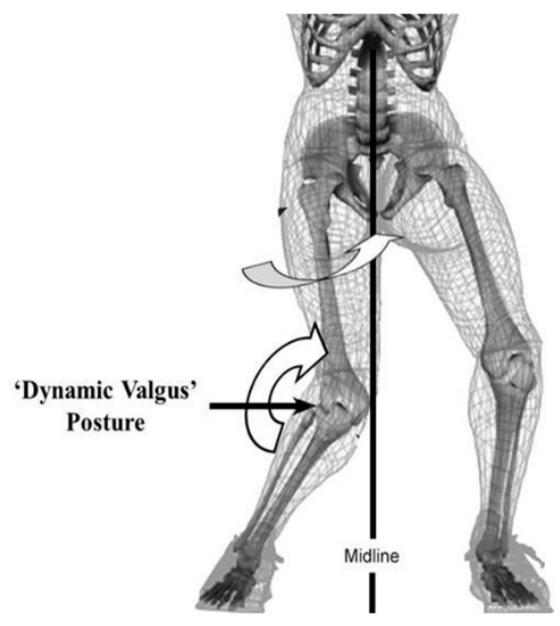

Figure 5. High-risk 'dynamic valgus' knee posture, which is a combination of hip internal rotation and abduction combined with knee flexion at impact.

\section{Delivery Principles}

As is illustrated above, researchers have made good progress toward understanding the components (i.e., content) that contribute to high-quality injury prevention training programs. It remains, though, that the efficacy of these programs is dependent in large part upon the extent to 
which they are (a) delivered effectively in the field, and (b) able to engage and motivate participants to adhere to them in the long-term. In recognition of these challenges, the BIT program draws from behaviour change and motivational theories to provide program instructors with the skills necessary to motivate those under their guidance and support adherence to the protocol.

Self-determination theory [6] is a widely-studied psychological framework that provides insight into the factors that support adaptive motivational responses. From a self-determination theory (SDT) standpoint, when individuals perceive an activity (such as an injury prevention program) to be enjoyable, personally relevant, and/or consistent with their values and identity, they experience autonomous (i.e., higher-quality) motivation. On the other hand, when an activity is pursued primarily due to a sense of obligation or because one is beholden to another (e.g., in the pursuit of external reward or the avoidance of punishment), individuals experience controlled (i.e., lower-quality) motivation. Autonomous forms of motivation been shown to underpin elevated engagement and positive behavioral outcomes [6], and as a result, it is noteworthy that SDT also outlines the factors (or 'needs') that support the development of this positive motivational orientation.

These needs relate to feeling a sense of autonomy (i.e., a sense of choice and volition), competence (i.e., feeling capable and having opportunities to achieved valued goals), and relatedness (i.e., feeling understood by and connected to others) in one's pursuits. SDT provides practical insights into the methods that may facilitate motivation and engagement, and accordingly, is widely utilised as an intervention framework in diverse contexts [33]. Guided by components within previous successful SDT-based trials [34,35], the BIT program purposefully teaches training principles that equip instructors with the knowledge and skill sets to support participants' needs for autonomy, competence, and relatedness. Autonomy support in a program such as the BIT intervention can be provided by (a) educating participants regarding the intended benefits of the program, (b) providing rationale(s) for key components within the program, and (c) allowing choice, inviting feedback, and soliciting participant input into the completion of core activities (i.e., encouraging participants to tailor their program in a way that align with the empirical evidence supporting the training recommendations). The provision of competence support can be achieved through, among other things, providing instructors with schedules for reviewing player progress, integrating goal-setting activities into the program, and training instructors in the provision of positive feedback that emphasises learning, development, and improvement. An emphasis on structuring activities to allow for gradual progression is also important in this respect, as are methods for identifying and overcoming potential barriers and challenges that may arise within the program. Finally, the provision of relatedness support in programs such as the BIT intervention can be achieved by training instructors to be empathetic, provide individualised attention to recipients when needed, and solicit, listen to, and act upon recipient feedback. Instructors also have an important role to play in supporting relatedness perceptions by encouraging group participation and creating a sense of cohesion and support among participants. By incorporating the motivational principles outlined above (alongside the biomechanically-informed content), those delivering injury prevention programs (such as the BIT program) may be better able to support participants' experiences in the program (e.g., engagement and adherence). These adaptive responses, in turn, are likely to maximise program efficacy and participant outcomes in both the short and long term.

Behavior change techniques can be utilised to augment the motivational strategies outlined above. Practitioners are advised to consult taxonomies of strategies that can be utilised by clinicians [24] or are self-enactable by participants [36]. Customisation of behavior change techniques within the BIT program will be maximised when informed by a behavioral analysis of one's capability (physical and psychological), opportunity (physical and social), and motivation (reflective and automatic) to perform key behaviors [5]. This behavior analysis can be conducted via participant interviews and/or brief questionnaires [36], with the goal to clarify key barriers and enablers. For example, one may have the physical capabilities required to perform body weight-based exercises, yet may have limited psychological capabilities (e.g., remembering to complete prescribed exercises and how to correctly 
complete them; how best to manage or change their own behavior). In such cases, practitioners can introduce or define stimuli (e.g., text messages, Post-it notes) that serve the purpose of prompting the person to perform the behavior at prescribed times, and complete goal-setting activities to provide focus and structure for their efforts. Most people are familiar with the SMART goals acronym (specific, measurable, authentic, realistic, timed), but less so on the importance of action and coping plans to complement these behavior targets by detailing specifics of the performance of BIT (e.g., the Ws and how) and analysing potential obstacles to their execution and how best to mitigate them. Even one of the hallmarks of a motivationally-enriched communication style - providing rationales for activities—can be complemented by knowledge of behavior change techniques; for example, clinicians can provide clients with information about the health (e.g., reduce their risk of injury, strengthen endurance) and social and environmental consequences (e.g., supporting teammates through group exercises) of BIT as a means by which to help people internalise the value or intended purpose of the exercises. Practitioners can also establish social support networks around program participants to provide informational, practical, and emotional support in the attainment of program goals. We encourage readers to review these taxonomies of behaviour change techniques to consider how they can integrate them as part of BIT in their own practice. This process, we argue, will lead to improved and effective translation of this program among a broad spectrum of athletic populations.

\section{Real World Application}

In collaboration with the Australian Women's Hockey Team, BIT was implemented alongside their 2012-2013 training schedule, four sessions/week, $20 \mathrm{~min} /$ day. Immediate outcomes (i.e., following nine weeks) from the intervention saw 'high-risk' athletes reduce their peak valgus knee moments and subsequent knee and ACL injury risk predisposition by $29 \%(p=0.003)$ [37]. Following prolonged exposure (i.e., following 25 weeks) [37], the entire training group reduced their peak valgus knee moments and knee and ACL injury risk by $26 \%(g=0.30)$. Interestingly, in 2011, prior to the training intervention, there were 155 incidences of lower limb injuries, with three to the ACL. In 2012 and 2013, which was during of the intervention period, there were 124 and 48 incidences of lower limb injuries, respectively, with 0 to the ACL. An unexpected, but interesting, finding from their preseason training results following the first year of training was their athletic performance increased. Their endurance improved by $2.2 \%(p=0.002)$, their max speed test improved by $1.7 \%$, while maintaining their maximum strength. The findings are important as there is a misconception in the literature that athletic performance suffers when injury prevention research is implemented.

BIT was also implemented as a clinical control trial among community level female hockey players (two sessions/week, 15-20 min/day). Acute outcomes from the intervention (i.e., nine weeks) showed that the comparison group displayed moderate-to-large increases in both peak valgus $(\Delta+27 \%$, $d=-0.36)$ and internal rotation $(\Delta+38 \%, d=-0.56)$ knee moments, with negligible-to-no changes observed within the intervention group [38]. These training results suggest that adjunct BIT maintained an athlete's relative risk of lower limb and ACL injury over the training season, whereas the comparison group were at a greater risk of lower limb and ACL injury after a season of play. These training outcomes are also consistent with previous research [39,40], suggesting even low volume training (two 15-20 min sessions per week) can be used to mitigate the potential deleterious effects of regular season community-based sport participation.

From this pilot research, there is a rationale for the researchers to reconsider the training content and delivery methods of their prophylactic training interventions. Therein, researchers should shift their focus from the type of training prescribed (e.g., balance, plyometric, resistance) to the intended biomechanical focus of the training content (individual exercises), and incorporate established psychological and behavior change principles in their delivery methods to maximise training outcomes. 


\section{Summary}

With few successes in translating lower limb and ACL injury prevention research among heterogeneous athletic populations (i.e., adult males and females), there is a rationale for researchers to re-evaluate how to maximise prophylactic training outcomes. Through this process, BIT was developed, which incorporates the most recent empirical evidence and behavior change theories to best inform the training content and delivery methods of lower limb and ACL injury/re-injury prevention protocols.

After surveying relevant biomechanics and simulation research, the four pillars forging the content portion of biomechanically-informed training are to use body weight-based exercises, irrespective of the training genres (e.g., balance, plyometric, resistance), to improve athletes' 1) knee flexion dynamics [296, 2) trunk control 27], 3) gastrocnemius muscle strength [28], and 4) hip external rotator strength [29] during dynamic sporting tasks. Examples of trialed exercises can be found at www.youtube.com/bodyfitworkouts (Figure 6).

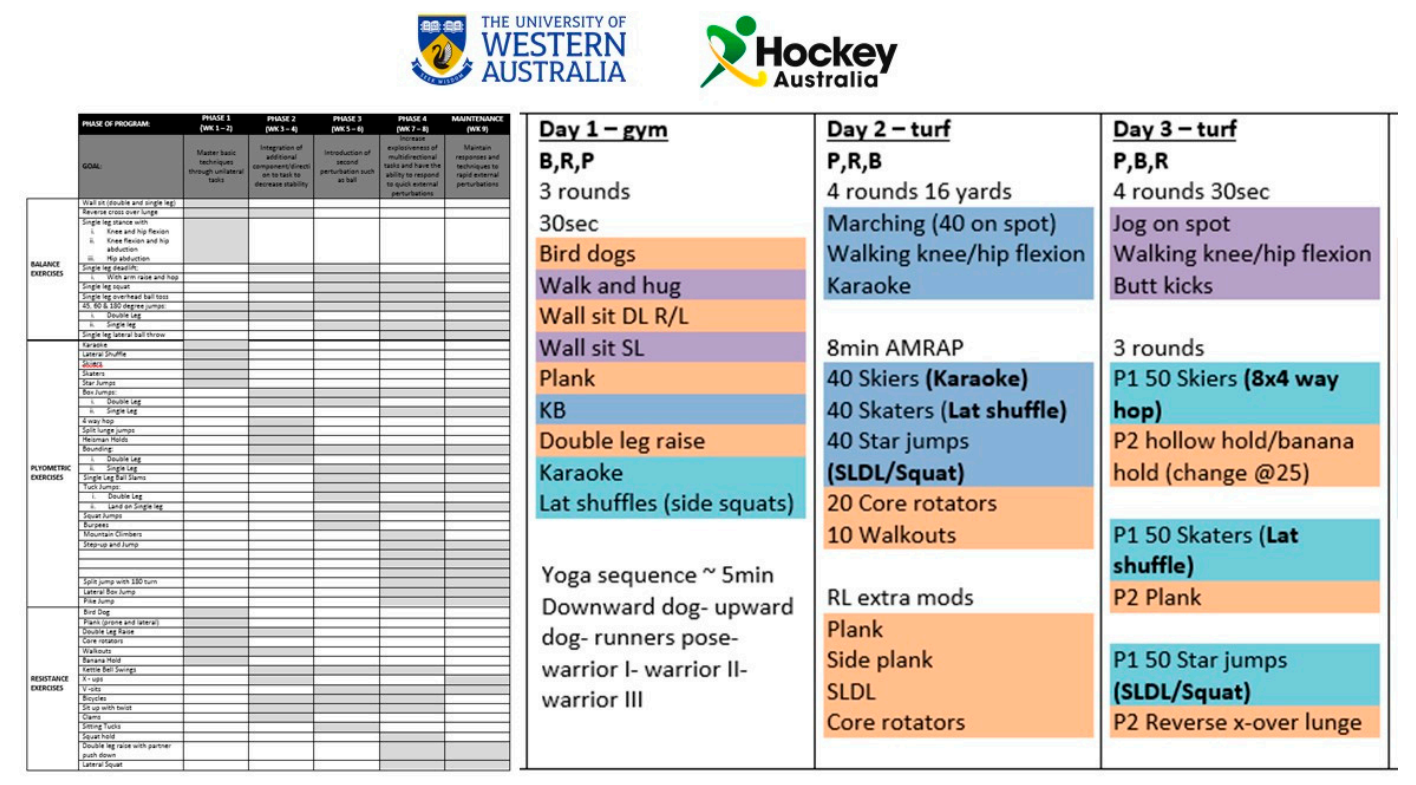

Figure 6. Example training program and schedule developed by The University of Western Australia in collaboration with Hockey Australia.

The psychological principles forming the foundation of the delivery methods of BIT is SDT, which involves autonomy (i.e., a sense of choice and volition), competence (i.e., feeling capable and having opportunities to achieve valued goals), and relatedness (i.e., feeling understood by and connected to others) [6].

BIT has been shown to have positive prophylactic effects among elite and community level female field hockey players alike. Future research is recommended to establish BIT as an effective prophylactic training approach for reducing lower limb and ACL injury rates among heterogeneous groups of athletes (sporting codes, skill levels, gender, age groups, etc.) worldwide.

Author Contributions: Conceptualization, C.J.D., J.R., B.S.J., and D.F.G.; biomechanics-focused methodology, C.D. and J.R.; psychology-focused methodology, B.J. and D.G.; writing and review editing, C.D., J.R., B.J., and D.G. All authors have read and agreed to the published version of the manuscript.

Funding: This research received no external funding.

Acknowledgments: We would like to thank all of the graduate students and colleagues for useful discussions over the years and for making this research possible. Special thanks to Gillian Weir, Jonathan Staynor, Joanna Nicholas, and Kristin Morgan for their fantastic experimental and physics based simulation based research contributions. We would also like to thank Josh "coach" Armstrong for help developing the training program. Lastly, we would also like to thank all of our participants for their time.

Conflicts of Interest: The authors declare no conflict of interest. 


\section{References}

1. Muybridge, E. The Science of the horse's Motion. Sci. Am. 1878, 39, 241.

2. Elfman, H. The measurement of the external forces in walking. Science 1938, 88, 152-153. [CrossRef]

3. Wolf, S. Handbook of Human Motion; Müller, B., Wolf, S.I., Brüggemann, G.-P., Deng, Z., McIntosh, A.S., Miller, F., Selbie, W.S., Eds.; Springer: Berlin/Heidelberg, Germany, 2018; ISBN 978-3-319-14417-7.

4. Sheeran, P.; Klein, W.M.P.; Rothman, A.J. Health behavior change: Moving from observation to intervention. Annu. Rev. Psychol. 2017, 68, 573-600. [CrossRef] [PubMed]

5. Michie, S.; Van Stralen, M.M.; West, R. The behaviour change wheel: A new method for characterising and designing behaviour change interventions. Implement. Sci. 2011, 6, 42. [CrossRef]

6. Ryan, R.M.; Deci, E.L. Self-determination theory and the facilitation of intrinsic motivation, social development, and well-being. Am. Psychol. 2000, 55, 68. [CrossRef]

7. Finch, C.F.; Kemp, J.L.; Clapperton, A.J. The incidence and burden of hospital-treated sports-related injury in people aged 15+ years in Victoria, Australia, 2004-2010: A future epidemic of osteoarthritis? Osteoarthr. Cartil. 2015, 23, 1138-1143. [CrossRef]

8. Gianotti, S.M.; Marshall, S.W.; Hume, P.A.; Blunt, L. Incidence of anterior cruciate ligament injury and other knee ligament injuries: A national population-based study. J. Sci. Med. Sport 2009, 12, 622-627. [CrossRef]

9. Janssen, K.W.; Orchard, J.W.; Driscoll, T.R.; van Mechelen, W. High incidence and costs for anterior cruciate ligament reconstructions performed in Australia from 2003-2004 to 2007-2008: Time for an anterior cruciate ligament register by Scandinavian model? Scand. J. Med. Sci. Sports 2012, 22, 495-501. [CrossRef] [PubMed]

10. Strehl, A.; Eggli, S. The value of conservative treatment in ruptures of the anterior cruciate ligament (ACL). J. Trauma 2007, 62, 1159-1162. [CrossRef] [PubMed]

11. Hartigan, E.H.; Axe, M.J.; Snyder-Mackler, L. Time line for noncopers to pass return-to-sports criteria after anterior cruciate ligament reconstruction. J. Orthop. Sport Phys. Ther. 2010, 40, 141-154. [CrossRef] [PubMed]

12. Orchard, J.; Seward, H.; McGivern, J.; Hood, S. Intrinsic and extrinsic risk factors for anterior cruciate ligament injury in Australian footballers. Am. J. Sports Med. 2001, 29, 196-200. [CrossRef]

13. Dunn, W.R.; Spindler, K.P.; MOON Consortium. Predictors of Activity Level 2 Years after Anterior Cruciate Ligament Reconstruction (ACLR): A Multicenter Orthopaedic Outcomes Network (MOON) ACLR Cohort Study. Am. J. Sports Med. 2010, 38, 2040-2050. [CrossRef]

14. Roos, H.; Ornell, M.; Gardsell, P.; Lohmander, L.S.; Lindstrand, A. Soccer after anterior cruciate ligament injury-An incompatible combination? A national survey of incidence and risk factors and a 7-year follow-up of 310 players. Acta Orthop. Scand. 1995, 66, 107-112. [CrossRef]

15. Donnelly, C.J.; Elliott, B.C.; Ackland, T.R.; Doyle, T.L.A.; Besier, T.F.; Finch, C.F.; Cochrane, J.L.; Dempsey, A.R.; Lloyd, D.G. An anterior cruciate ligament injury prevention framework: Incorporating the recent evidence. Res. Sports Med. 2012, 20, 239-262. [CrossRef] [PubMed]

16. Hewett, T.E.; Lindenfeld, T.N.; Riccobene, J.V.; Noyes, F.R. The effect of neuromuscular training on the incidence of knee injury in female athletes. A prospective study. Am. J. Sports Med. 1999, 27, 699-706. [CrossRef]

17. Mandelbaum, B.R.; Silvers, H.J.; Watanabe, D.S.; Knarr, J.F.; Thomas, S.D.; Griffin, L.Y.; Garrett, W., Jr. Effectiveness of a neuromuscular and proprioceptive training program in preventing anterior cruciate ligament injuries in female athletes: 2-year follow-up. Am. J. Sports Med. 2005, 33, 1003-1010. [CrossRef] [PubMed]

18. Finch, C.F. No longer lost in translation: The art and science of sports injury prevention implementation research. Br. J. Sports Med. 2011, 45, 1253-1257. [CrossRef] [PubMed]

19. Myklebust, G.; Engebretsen, L.; Braekken, I.H.; Skjolberg, A.; Olsen, O.E.; Bahr, R. Prevention of anterior cruciate ligament injuries in female team handball players: A prospective intervention study over three seasons. Clin. J. Sport Med. 2003, 13, 71-78. [CrossRef] [PubMed]

20. Junge, A.; Rosch, D.; Peterson, L.; Graf-Baumann, T.; Dvorak, J. Prevention of soccer injuries: A prospective intervention study in youth amateur players. Am. J. Sports Med. 2002, 30, 652-659. [CrossRef]

21. Soderman, K.; Werner, S.; Pietila, T.; Engstrom, B.; Alfredson, H. Balance board training: Prevention of traumatic injuries of the lower extremities in female soccer players? A prospective randomized intervention study. Knee Surg. Sports Traumatol. Arthrosc. 2000, 8, 356-363. [CrossRef] 
22. Steffen, K.; Myklebust, G.; Olsen, O.E.; Holme, I.; Bahr, R. Preventing injuries in female youth football-A clusterrandomized controlled trial. Scand. J. Med. Sci. Sports 2008, 18, 605-614. [CrossRef]

23. Ntoumanis, N.; Ng, Y.Y.; Prestwich, A.; Quested, E.; Hancox, J.E.; Thøgersen-Ntoumani, C.; Deci, E.L.; Ryan, R.M.; Lonsdale, R.M.; Williams, G.C. A meta-analysis of self-determination theory-informed intervention studies in the health domain: Effects on motivation, health behavior, physical, and psychological health. Health Psychol. Rev. 2020, 1-31. [CrossRef] [PubMed]

24. Ntoumanis, N.; Quested, E.; Reeve, J.; Cheon, S.H. Need supportive communication: Implications for motivation in sport, exercise, and physical activity. In Persuasion and Communication in Sport, Exercise, and Physical Activity; Jackson, B., Dimmock, J.A., Compton, J., Eds.; Routledge: Abingdon, UK, 2018; pp. 155-169.

25. Teixeira, P.J.; Marques, M.M.; Silva, M.N.; Brunet, J.; Duda, J.; Haerens, L.; La Guardia, J.; Lindwall, M.; Londsdale, C.; Markland, D.; et al. Classification of techniques used in self-determination theory-based interventions in health contexts: An expert consensus study. Motiv. Sci. 2019. [CrossRef]

26. Robinson, M.A.; Donnelly, C.J.; Vanrenterghem, J.; Pataky, T.C. Sagittal plane knee kinematics predict non-sagittal knee joint moments in unplanned sidestepping. In Proceedings of the XXV Congress of the International Society of Biomechanics, Glasgow, UK, 12-16 July 2015.

27. Donnelly, C.J.; Elliott, B.; Lloyd, D.G.; Reinbolt, J.A. Optimizing Whole body Kinematics to minimize valgus knee loading during sidestepping: Implications for ACL injury risk. J. Biomech. 2012, 45, 1491-1497. [CrossRef] [PubMed]

28. Morgan, K.; Donnelly, C.J.; Reinbolt, J.A. Elevated Gastrocnemius Forces Compensate for Decreased Hamstrings Forces during the Weight-Acceptance Phase of Single-Leg Jump Landing: Implications for Anterior Cruciate Ligament Injury Risk. J. Biomech. 2014, 47, 3295-3302. [CrossRef] [PubMed]

29. Hewett, T.E.; Myer, G.D.; Ford, K.R.; Heidt, R.S., Jr.; Colosimo, A.J.; McLean, S.G.; van den Bogert, A.J.; Paterno, M.V.; Succop, P. Biomechanical measures of neuromuscular control and valgus loading of the knee predict anterior cruciate ligament injury risk in female athletes: A prospective study. Am. J. Sports Med. 2005, 33, 492-501. [CrossRef]

30. Pataky, T.C.; Vanrenterghem, J.; Robinson, M.A. Zero- vs. one-dimensional, parametric vs. non-parametric, and confidence interval vs. hypothesis testing procedures in one-dimensional biomechanical trajectory analysis. J. Biomech. 2015, 48, 1277-1285. [CrossRef] [PubMed]

31. Delp, S.L.; Anderson, F.C.; Arnold, A.S.; Loan, P.; Habib, A.; John, C.T.; Guendelman, E.; Thelen, D.G. OpenSim: Open-source software to create and analyze dynamic simulations of movement. IEEE Trans. Biomed. Eng. 2007, 54, 1940-1950. [CrossRef]

32. Krosshaug, T.; Nakamae, A.; Boden, B.P.; Engebretsen, L.; Smith, G.; Slauterbeck, J.R.; Bahr, R. Mechanisms of anterior cruciate ligament injury in basketball: Video analysis of 39 cases. Am. J. Sports Med. 2007, 35, 359-367. [CrossRef]

33. Cochrane, J.L.; Lloyd, D.G.; Buttfield, A.; Seward, H.; McGivern, J. Characteristics of anterior cruciate ligament injuries in Australian football. J. Sci. Med. Sport 2007, 10, 96-104. [CrossRef]

34. Teixeira, P.; Marques, M.M.; Silva, M.N.; Brunet, J. A Classification of Motivation and Behavior Change Techniques Used in Self- Determination Theory-Based Interventions in Health Contexts. Motiv. Sci. 2020. [CrossRef]

35. Keyworth, C.; Epton, T.; Goldthorpe, J.; Calam, R.; Armitage, C.J. Acceptability, reliability, and validity of a brief measure of capabilities, opportunities, and motivations ("COM-B"). Br. J. Health Psychol. 2020. [CrossRef] [PubMed]

36. Knittle, K.; Heino, M.; Marques, M.M. The compendium of self-enactable techniques to change and self-manage motivation and behaviour v.1.0. Nat. Hum. Behav. 2020, 4, 215-223. [CrossRef] [PubMed]

37. Weir, G.; Alderson, J.; Elliott, B.C.; Lee, S.; Devaprakash, D.; Starre, K.; Cooke, J.; Goodman, C.; Armstrong, J.; Jackson, B.; et al. A two-year biomechanically informed ACL injury prevention program in female field hockey players. Transl. J. Am. Coll. Sports Med. 2019, 4, 206-214. 
38. Staynor, J.M.D.; Nicholas, J.C.; Weir, G.; Alderson, J.A.; Donnelly, C.J. Targeting associated mechanisms of anterior cruciate ligament injury in female community-level athletes. Sports Biomech. 2017, 16, 501-513. [CrossRef] [PubMed]

39. Donnelly, C.J.; Elliott, B.C.; Doyle, T.L.A.; Finch, C.F.; Dempsey, A.R.; Lloyd, D.G. Changes in muscle activation following balance and technique training and a season of Australian football. J. Sci. Med. Sport 2015, 18, 348-352. [CrossRef]

40. Donnelly, C.J.; Elliott, B.C.; Doyle, T.L.A.; Finch, C.F.; Dempsey, A.R.; Lloyd, D.G. Changes in knee joint biomechanics following balance and technique training and a season of Australian football. Br. J. Sports Med. 2012, 46, 917-922. [CrossRef]

(C) 2020 by the authors. Licensee MDPI, Basel, Switzerland. This article is an open access article distributed under the terms and conditions of the Creative Commons Attribution (CC BY) license (http://creativecommons.org/licenses/by/4.0/). 